\title{
Optical data storage in photochromic compounds
}

\author{
William Dallari ${ }^{\mathrm{a}, \mathrm{b}}$, Marco Scotto d'Abbusco ${ }^{\mathrm{a}}$, Elena Samoylova ${ }^{\mathrm{a}}$, Francesca Pignatellia, Athanassia Athanassiouc ${ }^{\mathrm{c}}$, \\ Roberto Cingolani ${ }^{\mathrm{a}}$, Alberto Diaspro, ${ }^{\mathrm{a}, \mathrm{b}}$, Marco Allione ${ }^{\mathrm{a}}$ \\ a Italian Institute of Technology, via Morego 30, I-16163, Genoa, Italy; \\ ${ }^{b}$ Department of Physics, University of Genoa, via Dodecaneso 33, I-16146, Genoa, Italy; \\ ${ }^{c}$ Center for Biomolecular Nanotechnologies - Italian Institute of Technology, via Barsanti 1, I-73010, Arnesano, \\ Italy.
}

\begin{abstract}
The future of optical data storage and the search for next generation high-density technologies have become a topic of lively debate. Three dimensional (3D) optical data storage, a relatively new technique which enables hundredslayer data recording in a hard memory, seems a viable candidate for this role. In this work, we report on 3D optical data storage in photochromic compounds. The recording medium used in the experiments was a photochromic diarylethenes derivative embedded in different host polymers. Multi-photon processes capability of triggering photochemical reactions and physical changes with micrometer-sized resolution in three dimensions offers a wellsuited means to achieve volumetric storage densities. A commercial microscope coupled to pulsed NIR and CW lasers have been used to write, read and erase data in our prototype memories. Information was recorded within the volume of the media via a multi-photon process achieving high localized photoconversion and the fluorescence emission from the photoconverted molecules when excited by a $405 \mathrm{~nm}$ laser diode was the readout signal. It is also shown that data recorded in such a medium can be selectively erased by irradiating with $514 \mathrm{~nm} \mathrm{CW}$ laser light giving the chance to write new data in the formerly used memory space. These storage media, thus, have good potentials to achieve volumetric storage of data although they certainly deserve further optimization work to achieve high number of superposed layers and to assure durability of the written data under readout conditions.
\end{abstract}

Keywords: photochromic molecules, optical data storage, multi-photon confocal microscopy.

\section{INTRODUCTION}

Despite the global recession, the amount of digital information created in 2009 grew $62 \%$ over 2008 to 0.8 Zettabytes (one ZB equals $10^{21}$ B) and is expected to grow by a factor of 44 from 2009 to 2020 , as all nations complete the transition from analog to digital [1]. This information overload is having a huge impact on data storage. In 2007, for the first time, the amount of produced digital information exceeded the amount of available storage and so far that gap continues to widen [2]. Traditional optical storage technologies, in fact, reached their data density limits and cannot address the overwhelming growth of digital information. Therefore, the development of ultrahigh capacity optical storage devices is mandatory. Recently, the first ever storage of 1TB of data on a DVD-sized disk using a storage technology based on recording data in three dimensions by means of Two-Photon Absorption (2PA) have been reported [3].

The recording principles behind this technology, called 2-Photon-3D, are based on the fact that, as two-photon processes are nonlinear and require high-optical intensities to occur, they typically only take place at the focal point of the laser beam. This characteristic offers the ability to store hundreds of layers within the volume of a DVD-type disk simply changing the focusing position of the writing beam within the recording medium. This micrometer-sized $3 \mathrm{D}$ resolution is at the base of 2-Photon-3D disks TB data capacities. In such systems data reading can be done activating the medium with a second laser and detecting the fluorescence of the recorded bits. Two-photon induced fluorescence-media are particularly advantageous because readout can be performed in parallel, i.e. it is possible to directly access to a plane of information. To summarize, 2-Photon-3D technology can offer higher storage capacities and data transfer rates than traditional storage media (such as DVD and Blu-ray disks) offer and seems able to meet future storage requirements. In this work, we report on 3D optical data storage in different photochromic materials

Organic Photonic Materials and Devices XIII, edited by Robert L. Nelson, François Kajzar, Toshikuni Kaino, Yasuhiro Koike, Proc. of SPIE Vol. 7935, 793510 - @ 2011 SPIE · CCC code: 0277-786X/11/\$18 · doi: 10.1117/12.879839 
obtained by mixing a photochromic diarylethenes derivative with different types of commercially available acrylic polymers. A comparison of the results obtained with the different matrices is also presented.

\section{MATERIALS AND METHODS}

Diarylethenes belong to the thermally irreversible group of photochromic compounds [4]. This property, added to their high resistance to fatigue which allows performing several write-read-erase cycles [5, 6], makes diarylethenesbased systems very attractive for optical data storage. The specific molecule that we utilized for recording information by 2PA was 1,2-Bis[2-methylbenzo[b]thiophen-3-yl]-3,3,4,4,5,5-hexafluoro-1-cyclopentene (MBT). This molecule undergoes cyclization/cycloreversion photochromic reactions upon light irradiation. Irradiation with UV light, in fact, leads to the formation of an aromatic ring via a $\mathrm{C}-\mathrm{C}$ bond [7]. This bond modifies the optical properties of the molecules and, in particular, its absorption spectrum, with the appearance of a broad absorption band in the visible, centered around $600 \mathrm{~nm}$, and of a fluorescence emission spectrum approximately in the same spectral range [8]. Vice versa, shining light in the visible portion of the spectrum causes a back-conversion of the molecule to the original colorless open form.

In order to study its behavior in a polymer matrix, we incorporated MBT molecules into different polymers. Our attention was mainly focused on Poly methyl methacrylate (PMMA), Poly ethyl methacrylate (PEMA), and Poly ethyl methyl methacrylate (PEMMA). Typically, 10wt\% MBT was mixed with $150 \mathrm{mg}$ of polymer and dissolved in $1 \mathrm{~mL}$ of solvent (toluene or chloroform). Thick films (up to $\approx 200 \mu \mathrm{m}$ ) were prepared by drop-casting on a microscope cover-slide. And finally, the sample was heated at $40^{\circ} \mathrm{C}$ (below polymers $\mathrm{Tg}$ ) for 1 hour in order to remove solvent residuals.

Optical data writing, reading, and erasing were performed using a commercial confocal microscope (Olympus FV1000, inverted) equipped with multiple laser lines: a Ti:Sapphire laser (Mai Tai, Spectra Physics) operating at 80 $\mathrm{MHz}, 720 \mathrm{~nm}$, and a pulse width of approximately $100 \mathrm{fs}$, a $405 \mathrm{~nm}$ laser diode and a multi-line $\mathrm{Ar}^{+}$laser tuned at $514 \mathrm{~nm}$. A water immersion objective (40x magnification, NA 0.90) focuses the laser beam into the specimen. The detection of a change in photoluminescence upon excitation from a CW laser source $\left(\lambda_{R}=405 \mathrm{~nm}\right)$ in the $575-640 \mathrm{~nm}$ range is used as readout signal. The green line of the $\mathrm{CW} \operatorname{Ar}^{+}$laser $\left(\lambda_{\mathrm{E}}=514 \mathrm{~nm}\right)$ was used to erase information in the recording medium.

\section{RESULTS AND DISCUSSION}

First step of our work was to demonstrate the localized activation of the photochemical reaction in the medium by 2PA. Figure 1 shows fluorescence images collected in the confocal microscope from an area irradiated by a single ultrafast femtosecond laser spot (see figure caption for technical details).

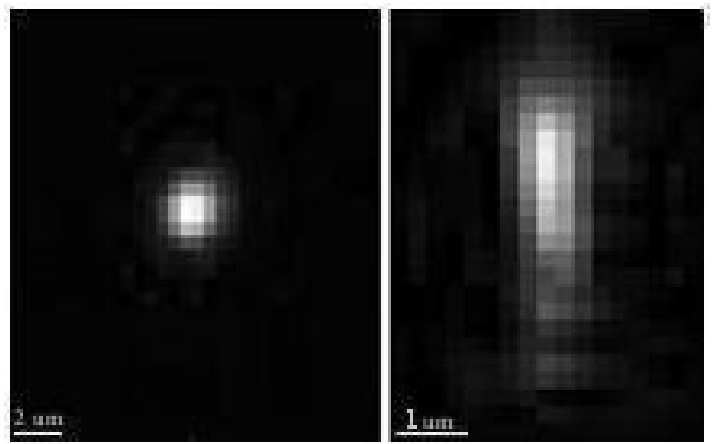

Figure 1. Lateral ( $x-y$, left) and axial ( $x-z$, right) profile of a diffraction-limited photoconverted voxel after 2PA.The sample is a blend of MBT in PEMMA prepared according to the procedure described in the text $\left(\lambda_{R}=405 \mathrm{~nm}\right.$, detection range $=575-640$ $\mathrm{nm})$. 
The emission detected in the 575-640 $\mathrm{nm}$ spectral range provides a good contrast between the recorded spot and the background enabling thus an easy retrieval of the stored bits. This is in agreement with measurements performed on the very same photochromic molecule in solution that showed that only molecules in the closed form present an emission spectrum in the red-orange spectral range, indicating that a photoconversion effectively took place in the spot area. The nonlinear behavior of the photoconversion process is shown in Figure 2. Different areas of the sample were irradiated with linearly growing values of the IR power used and single-photon $\left(\lambda_{R}\right)$ fluorescence images from each of these areas were collected at constant power. Then, the light intensity collected from each region of interest is plotted as function of the recording power. From the graph it is clear that there is a quadratic dependence of the fluorescence intensity on the IR power which proves the two-photon nature of the photoconversion process.

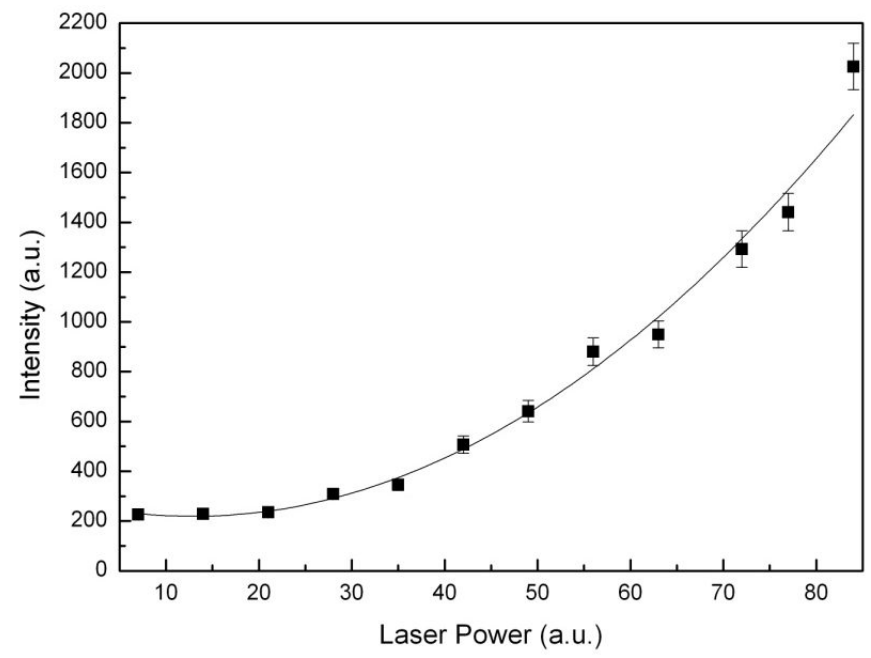

Figure 2. Photoluminescence intensity of selected areas as function of the laser intensity used for recording. The quadratic fit to the data $(\mathrm{R}=0.972)$ clearly demonstrate the nonlinear dependence of the signal and thus the multi-photon nature of the writing process.

In order to vertically overlap many layers of data without significant superposition, the spacing between adjacent layers must be sufficiently large. The interlayer spacing, actually, strictly depends on the threshold values set as logical ones or zeros. A good criterion to recognize the logical state, similar to the one used in electronics, is a 10$90 \%$ threshold-detection: the logical value " 1 " is associated to a recorded spot when the intensity signal is greater than $90 \%$ of the maximum value of the photoluminescence emitted from it, on the other hand, the logical value " 0 " is detected when the photoluminescence intensity is lower than $10 \%$ of the maximum value. In the hypothesis of a Gaussian beam profile in the vertical direction, the necessary spacing to avoid crosstalk can be easily estimated. Vertical spacing between layers must be approximately larger than 75\% of the FWHM of single peaks. For this value, the Gaussian tails of two nearest neighbors " 1 " logical values in the vertical direction give a residual signal in the intermediate " 0 " logical spot that remains lower than $10 \%$ of the peak values of the two adjacent bright spots. The same reasoning can be also applied to the spacing between adjacent spots within each single layer, rescaling it to the smaller size obtained in this case for the FWHM.

Figure 3 shows the intensity profile of the photoluminescence recorded in a sample of MBT-doped PEMA as function of the z-direction, i.e. the axis along which light propagates in the sample. In the same conditions as in Figure 1, single bits have been written in the sample equally spaced by $5 \mu \mathrm{m}$ in the $\mathrm{z}$ direction. The intensity profile shows that different layers of data, under these conditions, are well separated and single bits stored in different planes can be easily discriminated. The same measure, performed on samples of MBT doped PEMMA and PMMA are reported in Figure 4 and in Figure 5 respectively. Also in these cases as well it is clear that different layers are well separated one from the others. 


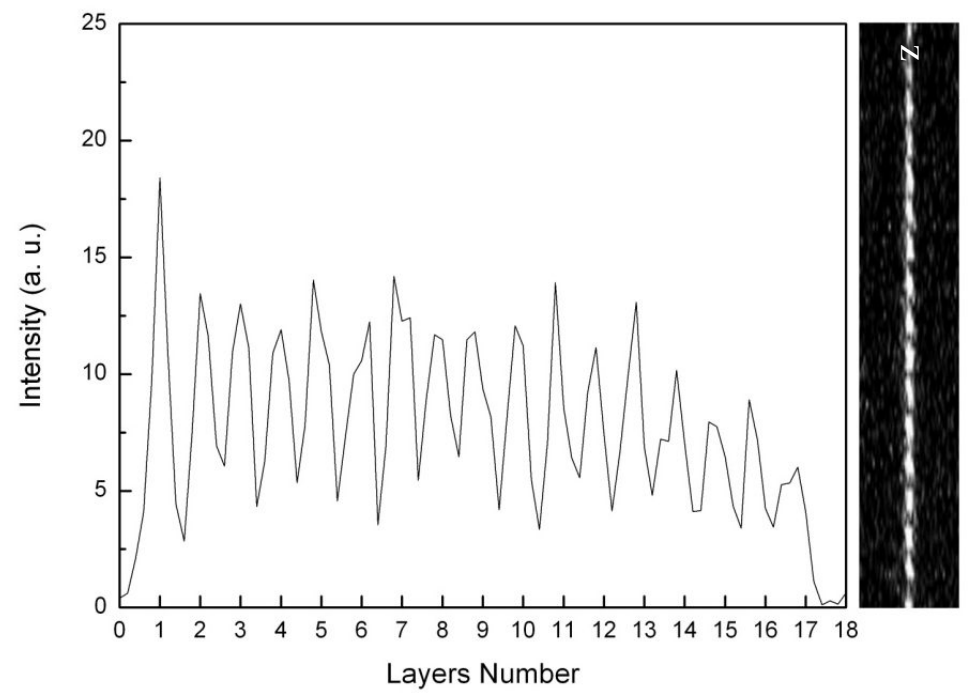

Figure 3. Intensity plot of the photoluminescence signal for a sequence of isolated spots aligned along the $\mathrm{z}$ direction. The sample is a blend of MBT in PEMA. The vertical displacement of the dots is $5 \mu \mathrm{m}$ while the conditions used to record the spots and to collect the photoluminescence are the same as in fig. 1 .

Moreover, our data indicate that the peak intensity of the obtained photoluminescence decreases along the $\mathrm{z}$ direction in all the samples. In order to provide an adequate explanation to this phenomenon, different factors must be taken into account. 3D optical data storage systems must record or access data at different depths in the medium, but a longer light path corresponds to a rising of scattering and aberration effects. These affect the focal shape and the intensity of the $\lambda_{R}$ laser and the pulsed IR beam, limiting then the efficiency of the reading and writing process. In addition, pulse duration of the IR femtosecond laser broadens when propagating through a dispersive medium, causing a further decrease of the efficiency of the writing process.

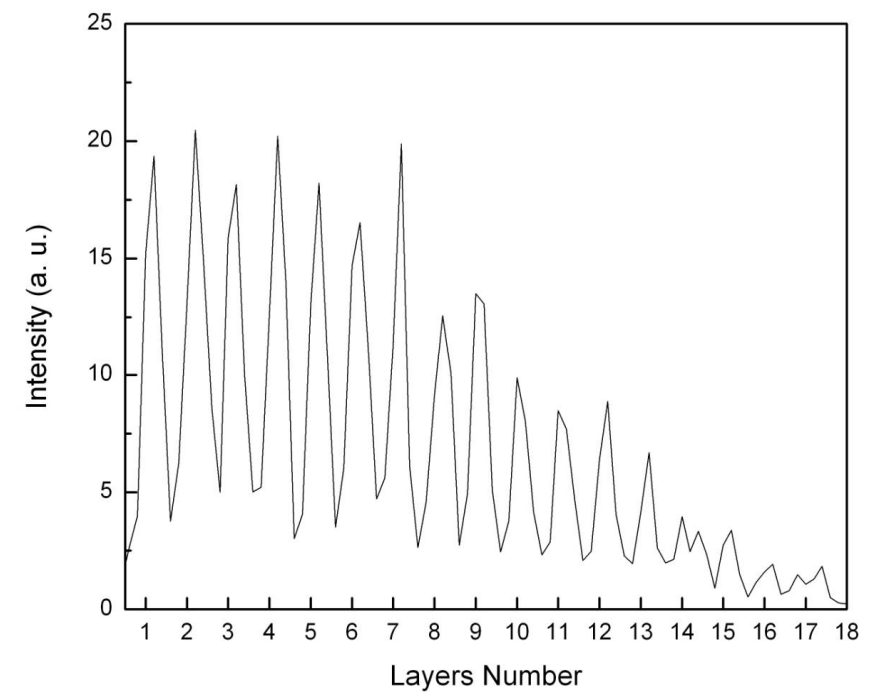

Figure 4. Same plot as in fig 3, but for a sample of MBT doped PEMMA. 


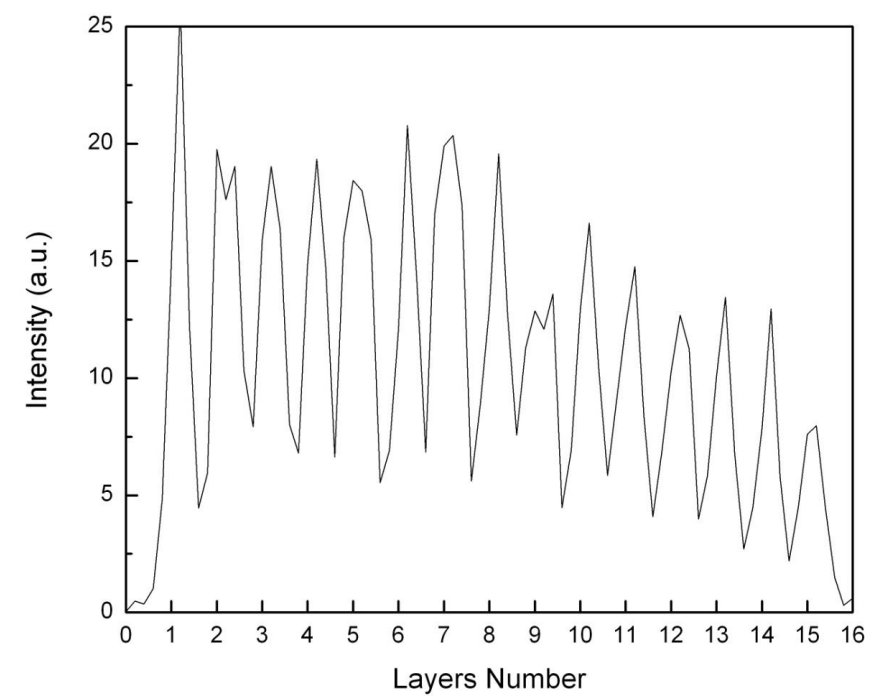

Figure 5. Same as in Figure 3 and Figure 4 but here the host polymer is PMMA.

As a consequence, the decrease in photoluminescence reported in the three graphs has to be attributed to the combination of the above-mentioned phenomena. It is also worth noting that the writing process is sensitive to the dye concentration. Relative small variations of molecules concentration lead to small fluctuations of the peak values. A decreased focusing capability affects as well the 3D spatial confinement of the spot. As it is evident from the three figures, the width of each single peak increases with depth. This qualitative consideration is quantified in Figure 6, where the FWHM of each photoluminescence spot is plotted as function of its $\mathrm{z}$ coordinates into the storage medium for the three different types of media used.

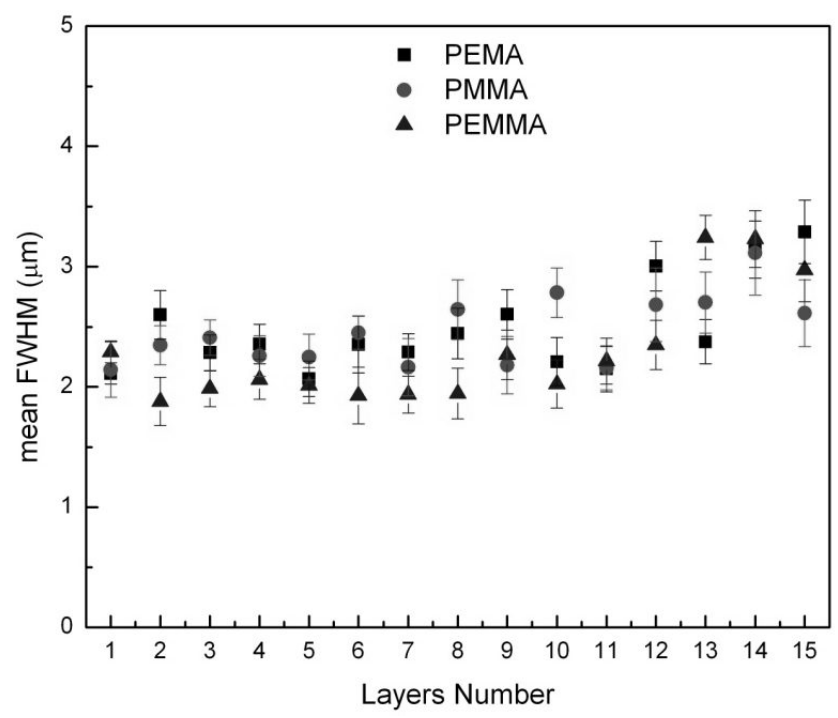

Figure 6. FWHM of isolated spots aligned along the $\mathrm{z}$ direction for MBT doped samples in the three different host polymers used, as indicated in the legend.

From Figure 6 it is then clear that axial FWHM shows a growing trend as function of $\mathrm{z}$, as a consequence of the above-mentioned processes. Optimizing the focusing optics could be a good method to circumvent this problem. Another solution could be to increase the interlayer spacing. Nevertheless, if high volumes of data have to be stored, hundreds of vertically-spaced layers are necessary and up to a certain extent the problem will certainly persist. With 
regard to the decrease in intensity of the signal emitted from recorded bits, it can be compensated by increasing the intensity of the recording and/or reading lasers.

Another important aspect is represented by molecule-matrix interactions, i.e., how the host polymer in which MBT molecules are embedded affects photochromic properties. It is reported in literature that the flexibility of the polymer chains in the vicinity of the photochromic molecules defines the speed at which the isomerization process takes place, i.e., the switching speed [9]. The molecular weight is an important parameter as well because it determines the mechanical properties of the polymer material and temperatures for transitions. In our case, chemical properties of the three different polymers are not expected to change much one from the other, since their structure is similar. On the other hand, their thermal and mechanical properties change, with a glass transition temperature ranging from 35$40{ }^{\circ} \mathrm{C}$ for the softer one (PEMA) up to above $100{ }^{\circ} \mathrm{C}$ for the harder one (PMMA). Furthermore, glass transition temperature seems to strongly affect the mobility of the molecules inside the polymer matrix during photoconversion processes and, hence, the photoswitching efficiency as well as it has been demonstrated to affect the fatigue resistance properties of the device [10].

\section{CONCLUSIONS}

In conclusion, we reported on the characterization of different media for application in 3D optical data storage. Storage matrices employed in our experiments were obtained by mixing transparent polymers with a photochromic molecule in a solution that was subsequently drop-cast on a glass support.

We tested the possibility to write, read and erase single digital bits into the material, associating each bit to a single laser spot inside the material volume, with the two logical stated determined in each spot by the two isomers of the photochromic molecules. Writing was carried out by a two-photon excitation process at the laser spot, coupling a near infrared femtosecond laser to an inverted confocal microscope. Readout was employed collecting photoluminescence emitted from molecules in the B form when excited with $405 \mathrm{~nm}$ wavelength laser light. It was demonstrated, in fact, that two-photon induced conformational change in the photochromic molecule causes the appearance of fluorescence emission in the 575-640 nm spectral range used for discriminating the two logical states of the digital memory. We demonstrated that in this geometry we can superpose many independent layers of data in the same device. Single bit spot was shown to be practically diffraction-limited with a FWHM of the order of few microns in the laser propagation direction and with a well evident signal standing out from the background. Our tests showed also that the spot size in the deepest data layers tends to broaden and that the peak intensity of the photoluminescence spots tends to decrease. These two effects can be attributed to the loss of efficiency in the twophoton process due to femtosecond pulse broadening that inevitably occurs when the pulse has to travel across an increasing thickness of dispersive medium and to the loss in best focusing conditions due to an increased aberration introduced in the system as far as the focus was moved deeper into the sample.

The possibility to erase independently stored data in the same confocal geometry by using laser light with $514 \mathrm{~nm}$ wavelength and to write new data in the very same part of the sample was demonstrated as well. Similar results were found in the different polymers used as embedding matrix, PMMA, PEMMA and PEMA, suggesting that all of them could be in principle suitable as a host material. Nevertheless, a host matrix must fulfill different requirements and, even if suitable as writing substrate, it might not be optimal for the application. Materials with a very low glass transition temperature exhibit mechanical properties which are generally not suitable for any commercial applications (11), but harder matrices could prove to be fragile and brittle. Moreover, also the effect of the matrix on the photochromic transition has to be taken into account: matrices with different physical or chemical properties might affect the photoinduced reaction, leading to a too high or too low efficiency in either the writing or the erasing process. This might lead to system in which writing could prove too demanding in terms of power or of exposure time required or in which information can be too easily deleted. For this reason, studies on readouts number are currently in progress in our group.

Another important property certainly dependent on the host polymer is the fatigue resistance of the device, i.e. the number of times data can be erased and rewritten before the device efficiency degrades irremediably. In order to study the effect of these three polymers on our photochromic molecule a more complete study has been performed and it is going to be published in an independent paper (10). 


\section{REFERENCES}

1. Gantz, J., Reinse, D., "The Digital Universe Decade: Are You Ready?" EMC Corporation. 2010.

2. Ganz, J. "The Expanding Digital Universe." International Data Corporation (IDC). 2007.

3. Dvornikov, A. S., Walker, E. P. and Rentzepis, P. M. "Two-Photon Three-Dimensional Optical Storage Memory,"

J. Phys. Chem. A, 113, 13633-13644 (2009).

4. Irie, M., "Dyarilethenes for Memories and Switches," Chem. Rev., 100, 1685-1716 (2000).

5. Kawata, S., Kawata, Y., "Three-Dimensional Optical Data Storage Using Photochromic Materials," Chem. Rev., 100, 1777-1788 (2000).

6. Tian, H., Yang, S., "Recent progresses on diarylethene based photochromic switches," Chem. Soc. Rev., 33, 85-97 (2004).

7. Jun, C., et al., "Photochromic fluorescence switching from diarylethenes and its applications," J. Photoch. Photobio. C, 10, 111-129 (2009).

8. Kang, J., Kim, E., Kim, J., "All-optical switch and modulator using photochromic dye doped polymer waveguides," Opt. Mater.,21, 543-548 (2002).

9. Krongauz, V., [Environmental Effects on Organic Photochromic Systems] Deurr, H., Bouas-Laurent, H., Eds.; Elsevier Amsterdam, 40, 793-820 (1990).

10. Samoylova, E., et al, "Highly fatigue resistant photochromic material for 3D optical memory," in preparation. 11. Odian, G., [Principles of polymerization], John Wiley \& Sons, Hoboken, New Jersey, 32-36 (2004). 